\title{
The Prevalence of Metabolic Syndrome in Coronary Artery Disease Patients
}

\author{
Farzaneh Montazerifar ${ }^{\mathrm{a}}$, Ahmad Bolourib ${ }^{\mathrm{b}}$, Milad Mahmoudi Mozaffar ${ }^{\mathrm{c}}$, \\ Mansour Karajibanid, e
}

\begin{abstract}
Background: Metabolic syndrome (MetS) is a worldwide health problem, which is growing in Iranian adults. MetS is associated with risk of type 2 diabetes and coronary artery disease (CAD). In this study, we aimed to investigate the prevalence of MetS and its individual components in $\mathrm{CAD}$ patients.
\end{abstract}

Methods: This cross-sectional study was performed on 200 CAD patients who had undergone elective coronary angiography at the cardiology department. Anthropometric indices including waist circumference (WC) and body mass index were measured. Blood samples were obtained to determine glucose and lipid profile. MetS components were defined according to the modified Adult Treatment Panel III (ATP III) criteria.

Results: The prevalence of MetS among patients was 49.5\% (women: $55.9 \%$; men: $40.2 \%$; $\mathrm{P}<0.05$ ). The prevalence increased with age. The low high-density lipoprotein-cholesterol (low HDL-C) (84.8\%), high fasting blood glucose (high FBG) $(77.8 \%)$ and high WC $(75.8 \%)$ were the most prevalent risk factors in CAD patients with MetS.

Conclusions: Recent data indicate that the dyslipidemia, hyperglycemia and abdominal obesity are crucial predictors of MetS in CAD patients. Further prospective studies are recommended for more clarification.

Keywords: Prevalence; Metabolic syndrome; Coronary artery disease

\footnotetext{
Manuscript accepted for publication November 22, 2016

aPregnancy Health Research Center, Department of Nutrition, School of Medicine, Zahedan University of Medical Sciences, Zahedan, Iran

${ }^{b}$ Department of Cardiology, School of Medicine, Zahedan University of Medical Sciences, Zahedan, Iran

'School of Medicine, Zahedan University of Medical Sciences, Zahedan, Iran ${ }^{\mathrm{d}}$ Health Promotion Research Center, Department of Nutrition, School of Medicine, Zahedan University of Medical Sciences, Zahedan, Iran

${ }^{\mathrm{e}}$ Corresponding Author: Mansour Karajibani, Department of Nutrition, School of Medicine, Zahedan University of Medical Sciences, Zahedan, Iran.

Email: mkarajibani@yahoo.com
}

doi: https://doi.org/10.14740/cr507w

\section{Introduction}

Coronary artery disease (CAD) is one of the most common causes of death in the developed world [1]. The high prevalence of cardiac risk factors and associated morbidity have been reported in the Iranian adult population [2]. These risk factors have become known as the metabolic syndrome (MetS). MetS is a growing health problem in Iranian adults due to the alterations in lifestyle, low physical activity, the epidemic of obesity and insulin resistance (IR) $[2,3]$. MetS includes several cardiometabolic risk factors and is characterized by four essential components including intra-abdominal obesity, dyslipidemia, hypertension and impaired glucose tolerance $[2,4]$, and linked to a high risk of both type 2 diabetes and $\mathrm{CAD}$, and increased risk of cardiovascular events $[2,5$, 6]. The epidemiologic studies on MetS in Iran have revealed the high prevalence of MetS in the north of Iran (37.9\%) [4], Zahedan, southeast of Iran (28.5\%) [7], and in the province of Zanjan, the west of Iran (23.7\%) [8]. Based on the Tehran Lipid and Glucose Study, the prevalence of MetS in the Iranian adults was reported to be $33.7 \%$ (24\% for men vs. $42 \%$ for women) [9].

The association between MetS and its components with $\mathrm{CAD}$ has been reported in several studies using different techniques $[1,5,6,10]$. According to ATP III criteria, diagnosis of MetS is based on the presence of three or more of the five criteria including waist circumference (WC) $>102$ in men and $>88$ in women, high blood pressure (BP $\geq 130 / 85)$, high triglyceride ( $\mathrm{TG} \geq 150$ ), high fasting blood sugar ( $\mathrm{FBS} \geq 110$ ), and low high-density lipoprotein (HDL $<40$ in men and $<50$ in women) [11]. The American Heart Association (AHA) and National Health Lung and Blood Institute (NHLBI) modified the ATP III criteria with a reduction in FBS from 110 to 100 $\mathrm{mg} / \mathrm{dL}[3,12]$. As well, the Iranian National Committee of Obesity (INCO) considered cut-offs of $\geq 95 \mathrm{~cm}$ for both men and women in Iran, which could predict the incidence of cardiovascular disease (CVD) [3, 13].

Each of MetS components is considered as an independent cardiac risk factor and increased number of components elevates the severity of CVD [1]. Central fat distribution is a more atherogenic property than peripheral obesity [14], and WC is evaluated to identify the central obesity. Thus, abdominal obesity is considered as one of the components of MetS, which is linked to the occurrence of dyslipidemia, diabetes and 
Table 1. Demographic and Clinical Characteristics of in CAD Patients According to MetS

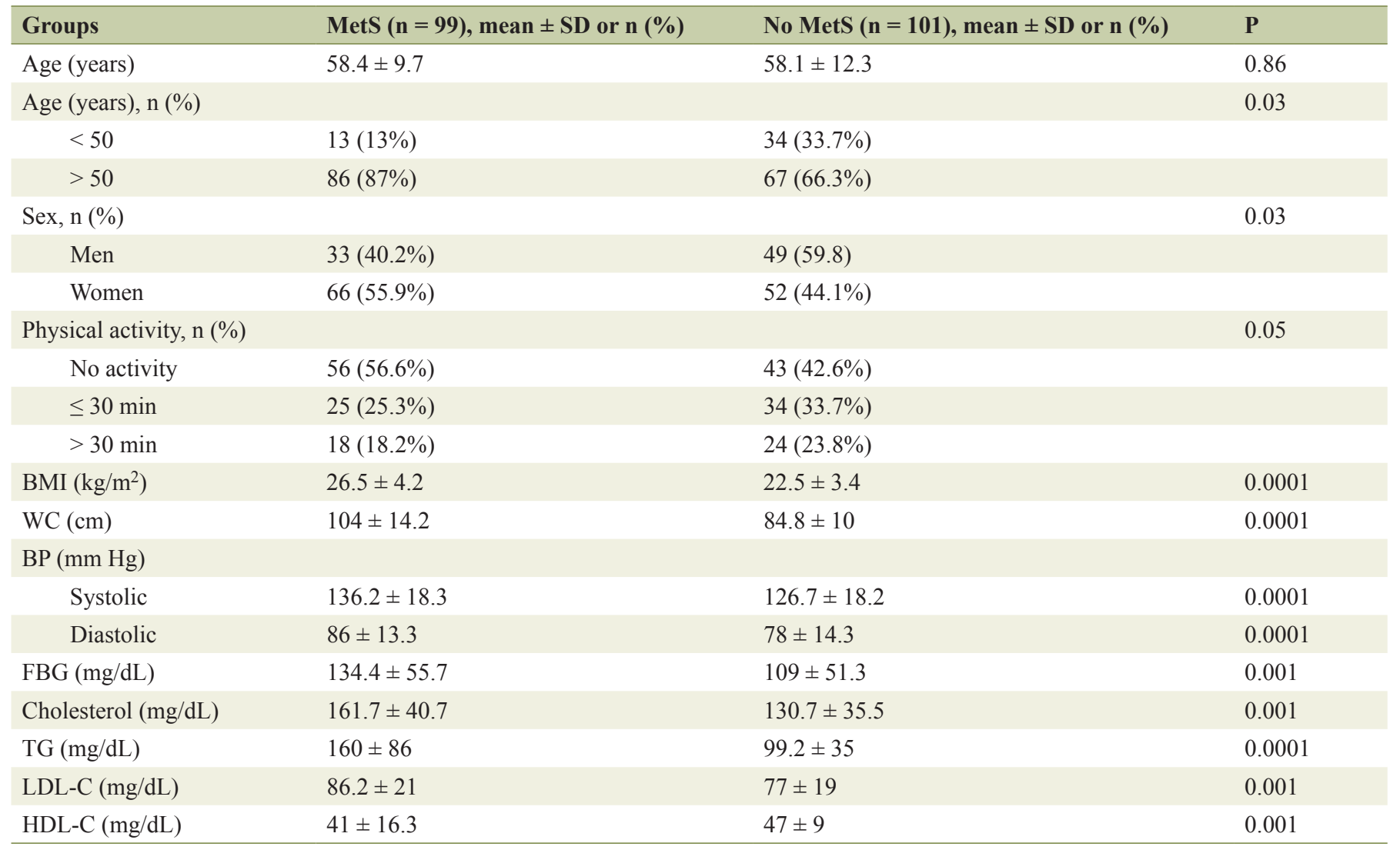

Data are expressed as mean \pm SD. MetS: metabolic syndrome; BMI: body mass index; WC: waist circumference; BP: blood pressure; FBG: fasting blood glucose; TG: triglyceride; LDL-C: low-density lipoprotein-cholesterol; HDL-C: high-density lipoprotein- cholesterol.

risk of cardiovascular events [3]. IR is also one of the major characteristics of MetS, and considerably affects the development of CAD $[5,10]$. In a study, low HDL, high fasting plasma glucose (high FPG), high BP and high TG were reported as the highest risk factors for coronary heart disease [15]. Based on the above-mentioned evidence, diagnosis of MetS and its components in patients with CAD can influence prevention of disease and management programs $[5,6,10]$. Thus, this study aimed to investigate the prevalence of MetS and its individual components in CAD patients who underwent elective coronary angiography.

\section{Patients and Methods}

This cross-sectional study was performed on 200 CAD patients (mean age $58.3 \pm 11.1$ years) admitted to Cardiology Department of Imam Ali Hospital in Zahedan, southeast of Iran, and diagnosed by a hospital cardiologist, during a 5-month period from June to October 2016.

CAD was diagnosed based on a combination of previous medical history, clinical findings (e.g., dyspnea, and excessive fatigue, and suspected ischemia), and electrocardiogram (ECG) changes, and confirmed with $50 \%$ or more coronary artery stenosis in at least one major coronary artery as assessed by coronary angiography.

The patients with chronic heart failure, myocardial infarction or unstable angina pectoris and acute coronary syndrome (ACS) who needed emergent coronary intervention or surgery, hepatic and kidney diseases, hyperthyroidism, pregnancy, and patients with ejection fraction $(\mathrm{EF})<60 \%$ were excluded from study.

All patients gave oral informed consent, and ethical approval was obtained from Ethics Committee of Zahedan University of Medical Sciences (approval date April 24, 2016, No. 1827: IR.ZAUMS.REC.1395.11). The demographic data including age, sex, physical activity, and previous medical history and medications were completed at the first meeting with patients. The age was categorized as $<50$ and $\geq 50$ years old. Physical activity was classified into three categories: no activity $<3$, and $\geq 30$ min walking for at least three times a week [16].

The weight and height of patients were recorded with light clothes and without shoes by a Seca scale (Germany) to the nearest $0.5 \mathrm{~kg}$ and $0.5 \mathrm{~cm}$, respectively. Body mass index (BMI) was calculated as weight $(\mathrm{kg}) /$ height $\left(\mathrm{m}^{2}\right)$.

WC was measured in the standing position using an inelastic measuring tape to the nearest $0.5 \mathrm{~cm}$ between the lower border of the rib cage and the iliac crest [2].

$\mathrm{BP}$ was measured in the seated position after at least $5 \mathrm{~min}$ 
of rest. The measurement was performed on the right arm using a digital manometer (model ALP K2, K2-231, Japan). Two recordings were carried out, and the mean levels of BP were used for analysis.

The blood samples were obtained after overnight fasting. Serum levels of total cholesterol, high-density lipoproteincholesterol (HDL-C), low-density lipoprotein- cholesterol (LDL-C), TG and fasting blood glucose (FBG) were assayed by enzymatic procedures (Pars Azmon Kit, Tehran, Iran) using an auto-analyzer (Hitachi, Japan).

In this study, MetS was described as the presence of $\geq 3$ of the following criteria, according to the modified protocol of Adult Treatment Panel (ATP III), with regional cut-off value of $W C \geq 95 \mathrm{~cm}$ for both gender, increased FBG $\geq 100 \mathrm{mg} / \mathrm{dL}$, hypertriglyceridemia (TG $\geq 150 \mathrm{mg} / \mathrm{dL})$, decreased HDL-C $(<$ $40 \mathrm{mg} / \mathrm{dL}$ in men or $<50 \mathrm{mg} / \mathrm{dL}$ in women), and hypertension (systolic blood pressure (SBP) $\geq 130 \mathrm{~mm} \mathrm{Hg}$ and diastolic blood pressure (DBP) $\geq 85 \mathrm{~mm} \mathrm{Hg}$ ) [3].

\section{Statistical analyses}

Statistical analyses were performed using Statistical Package for the Social Sciences version 21 (SPSS Inc., Chicago, IL, USA). Data were presented as mean \pm standard deviation (SD), frequencies and percentages. Chi-square and Student's $t$-test were used for statistical analysis. Odds ratios (ORs) and 95\% confidence intervals (CIs) were calculated for MetS and individual components of it. Multivariable linear regression model, adjusted for sex, was used to evaluate the association between variables. $\mathrm{P}<0.05$ was considered significant.

\section{Results}

The demographic and clinical characteristics of the $200 \mathrm{CAD}$ patients ( 82 men and 118 women; aged $24-81$ years) with and without MetS are presented in Table 1. Of these, $49.5 \%$ had MetS ( $\mathrm{n}=99,95 \%$ CI: $4.8-9.3)$, and it was $55.9 \%$ among women and $40.2 \%$ among men $(\mathrm{P}<0.05)$. The highest prevalence was present in patients aged $>50$ years $(87 \%, \mathrm{P}<0.05)$. The mean WC, BMI and BP $(\mathrm{P}<0.0001)$, serum levels of FBG and cholesterol $(\mathrm{P}<0.001)$, TG $(\mathrm{P}<0.0001)$ and LDL-C $(\mathrm{P}<$ $0.001)$ in CAD patients with MetS were significantly higher, and HDL-C levels $(\mathrm{P}<0.001)$ were significantly lower than in those without MetS.

As shown in Table 2, low HDL-C (84.8\% vs. $67.3 \%$, P $<0.01)$, high FBG $(77.8 \%$ vs. $29.7 \%, \mathrm{P}<0.0001)$, and high WC $(75.8 \%$ vs. $9.9 \%, \mathrm{P}<0.0001)$ were the most prevalent risk factors in CAD patients with MetS as compared to those without MetS. As well, the findings revealed that the ORs for main components of MetS in CAD patients with MetS were as follows: low HDL-C (OR: 12.65; 95\% CI: 5.3 - 20), high WC (OR: 6.7; 95\% CI: 3.7 - 12.1), high FBG (OR: 6.7; 95\% CI: 5.9 - 8.76), high TG (OR: 2.26; 95\% CI: 1.1 - 3.48), and high BP (OR: 1.35 ; 95\% CI: 1.02 - 1.8).

Table 3 demonstrates that after adjusting for sex, based on multivariable linear regression analysis, the high $\mathrm{WC}(\beta=$
0.63, $\mathrm{P}<0.0001)$, high $\mathrm{BP}(\beta=0.19, \mathrm{P}<0.05)$, high FBG $(\beta=$ $0.14, \mathrm{P}<0.01)$, high TG $(\beta=0.19, \mathrm{P}<0.01)$ and low HDL-C $(\beta=0.20, P<0.0001)$ were found as significant predictors of MetS in CAD patients.

\section{Discussion}

In our study, the prevalence of MetS in CAD patients was $49.5 \%$. The studies performed regarding the prevalence of MetS and/or associated risk factors in CAD patients have demonstrated that the rate of MetS in patients hospitalized for ACS was between $43 \%$ and $51 \%$ [17]. In a similar study [1], in which conventional angiography was performed in suspected CAD patients, $40.5 \%$ of patients had MetS based on the AHA/ NHLBI criteria.

The findings of this study demonstrate that the prevalence of MetS in CAD patients is relatively higher than other regions of the world. These differences could be due to definition of MetS by different criteria, cultural mores, lifestyle-related factors such as improper food habits, physical inactivity, stress and sample size in various investigations [18].

Different combinations of the components of MetS have various effects on CVD; however, each component acts as an independent risk factor for $\mathrm{CAD}$, and all of them interact synergistically, and thus lead to increased risk of CAD [1].

The prevalence of individual components of MetS with various rates in $\mathrm{CAD}$ patients has been reported in earlier studies $[1,2,17,19,20]$.

The abdominal obesity, dyslipidemia (high TG and low HDL-C) [1, 2, 21], hypertension [2] and hyperglycemia [2, 21] most often were reported as the MS components, and the severity of CAD increased with the number of components [2]. A study performed in Shanghai showed that among individual components of MetS, low HDL, high FBG, and high BP had the highest $\mathrm{OR}$ for coronary heart disease. These three parameters had significant increases in number of disease vessel [15]. The results of a study in Korean population showed that MetS was independently linked to coronary parameters including obstructive plaque and coronary artery calcium score in the non-diabetic CAD patients. Among the individual components of MetS, low HDL-C levels were markedly associated with CAD in these patients [10, 22]. A study in Tehran revealed that $78 \%$ of men and $80 \%$ of women $>20$ years old had at least one risk factor of CVD. The prevalence of high FBG, high BP, obesity, high total cholesterol, low HDL-C, and high TG was reported to be $9.8 \%, 20.4 \%, 14.4 \%, 19.3 \%, 32 \%$, and $5.3 \%$, respectively [23].

In the current study, the CAD patients with MetS had significantly higher levels of BMI, WC, BP, serum lipid profile, and FBG, and lower HDL-C levels; however, regression analysis revealed that the rate of different components of MetS, including singularly a much higher rate of low HDL-C, high blood glucose and high WC, with the highest OR, and a relatively high prevalence of high TG and high BP was found in patients with MetS than those without MetS.

Several studies have demonstrated that dyslipidemia is common in Iranian population $[24,25]$. The low HDL-C was 
Table 2. Prevalence of Individual Components of MetS in CAD Patients Based on Gender

\begin{tabular}{|c|c|c|c|}
\hline Parameters & MetS $(n=99)$, OR $(95 \%$ CI $)$ & No MetS $(n=101)$, OR $(95 \%$ CI $)$ & $\mathbf{P}$ \\
\hline High WC, n (\%) & & & 0.0001 \\
\hline No & $24(24.2 \%)$ & $91(90.1 \%)$ & \\
\hline \multirow[t]{2}{*}{ Yes } & $75(75.8 \%)$ & $10(9.9 \%)$ & \\
\hline & $6.7(3.7-12.1)$ & $0.27(0.19-0.38)$ & \\
\hline High BP, n (\%) & & & 0.047 \\
\hline No & $46(46.5 \%)$ & $62(61.4 \%)$ & \\
\hline \multirow[t]{2}{*}{ Yes } & $53(53.5 \%)$ & $39(38.6 \%)$ & \\
\hline & $1.35(1.02-1.8)$ & $0.75(0.58-0.98)$ & \\
\hline High FBG, n (\%) & & & 0.0001 \\
\hline No & $22(22.2 \%)$ & $71(70.3 \%)$ & \\
\hline \multirow[t]{2}{*}{ Yes } & $77(77.8 \%)$ & $30(29.7 \%)$ & \\
\hline & $6.7(5.9-8.76)$ & $0.31(0.22-0.48)$ & \\
\hline High TG, n (\%) & & & 0.0001 \\
\hline No & $37(37.4 \%)$ & $96(95 \%)$ & \\
\hline \multirow[t]{2}{*}{ Yes } & $62(62.6 \%)$ & $5(5 \%)$ & \\
\hline & $2.26(1.1-3.48)$ & $0.39(0.3-0.5)$ & \\
\hline Low HDL-C, n (\%) & & & 0.01 \\
\hline No & $15(15.2 \%)$ & $33(32.7 \%)$ & \\
\hline \multirow[t]{2}{*}{ Yes } & $84(84.8 \%)$ & $68(67.3 \%)$ & \\
\hline & $12.65(5.3-20)$ & $0.46(0.27-0.8)$ & \\
\hline
\end{tabular}

Data are expressed as n (\%). WC: waist circumference; BP: blood pressure; FBG: fasting blood glucose; TG: triglyceride; HDL: highdensity lipoprotein; MetS: metabolic syndrome; OR (95\% CI): odds ratio (95\% confidence interval).

presented as one of the most prevalent abnormalities [8] and the strongest factor that independently linked to CAD in Iranian population [26] and other countries [10, 22]. High TG has been known as the second most prevalent abnormality [8]. Some studies have shown that among the individual components of MetS, high level glucose was markedly associated with CAD. A study reported that the prevalence of CHD in the diabetic patients with MetS was significantly higher than in those without MetS [27]. The high prevalence of diabetes in the Iranian population with established CAD [26, 28], and patients undergoing elective coronary artery bypass grafts (CABG) [29] was reported in some studies. Other investigation also reported that the long-term diabetes is linked to development of atherosclerosis [30].

It has been clarified that hypertension as one of the components of MetS is increasing among the Iranian population $[28,31]$, in particular, in patients with confirmed CAD $[25,26$, 32]. Several cross-sectional investigations performed in Iran have shown that the incidence rate of hypertension was significantly higher in CAD patients compared to healthy population $[25,32]$ and in non-insulin-treated than insulin-treated type 2 diabetes mellitus [33]. It was higher in men than in women [28], and increased with older age [21, 33].

There is evidence that the presence of obesity/abdominal obesity and its relationship with CAD is more common than other components of MetS, and abdominal obesity has been identified as a central component of Mets [2], which has a strong relationship with hypertension, IR and dyslipidemia $[2,3,31]$. The coronary angiography findings in CAD patients in Isfahan also showed the patients who had more WC were at higher risk of heart disease [34]. In our study, the mean of both BMI and WC in patients with MetS was significantly higher than those without MetS; however, after adjusting of sex, based on multivariable linear regression analysis, a positive significant association was found only between WC and MetS, suggesting that the fat distribution in the abdomen is more atherogenic than peripheral obesity and may predict the risk of CVD [24].

The findings of our study showed that three of MetS components including low HDL-C, high blood glucose and abdominal obesity, were the strongest predictors of CAD; however, older age, physical inactivity and female gender had also predictive value. Although after adjusting of sex, there was no statistically significant relationship between the categories of age and physical activity with the MetS in the CAD patients; however, these variables were significantly higher among CAD patients with MetS than in those without MetS. Similar studies in Iranian adult population reported that all major components of MetS increase with aging [19, 35, 36], and a higher rate of MetS [7, 8, 19] and its components [21] has been reported in women compared with men in earlier studies. Karimi et al [29] demonstrated that the 
Table 3. Results of Multivariate Linear Regression Analysis Between MetS and the Coronary Risk Factors in CAD Patients

\begin{tabular}{|c|c|c|c|c|c|}
\hline \multirow{2}{*}{ Parameters } & \multicolumn{2}{|c|}{ Unstandardized coefficients } & \multirow{2}{*}{ Standardized coefficients beta } & \multirow{2}{*}{$\mathbf{t}$} & \multirow{2}{*}{$\mathbf{P}$} \\
\hline & B & Standard error & & & \\
\hline Older age & 0.003 & 0.003 & 0.07 & 1.239 & 0.217 \\
\hline High BMI & 0.012 & 0.009 & 0.107 & 1.316 & 0.190 \\
\hline High WC & 0.020 & 0.003 & 0.634 & 7.464 & 0.0001 \\
\hline High BP & 0.005 & 0.002 & 0.186 & 2.385 & 0.018 \\
\hline High FBG & 0.001 & 0.000 & 0.142 & 2.595 & 0.01 \\
\hline High cholesterol & 0.000 & 0.001 & 0.031 & 0.449 & 0.654 \\
\hline High TG & 0.001 & 0.000 & 0.187 & 3.211 & 0.002 \\
\hline High LDL & 0.001 & 0.001 & 0.033 & 0.584 & 0.560 \\
\hline Low HDL & 0.007 & 0.002 & 0.197 & 3.854 & 0.0001 \\
\hline
\end{tabular}

MetS: metabolic syndrome; BMI: body mass index; WC: waist circumference; BP: blood pressure; FBG: fasting blood glucose; TG: triglyceride; LDL: low-density lipoprotein; HDL: high-density lipoprotein. Dependent variable: MetS.

patients with CAD were more male with older age. Won et al [10] reported that US population over 50 years of age with MetS had higher CHD prevalence when compared to those without MetS.

It is clear that the fat tissue in women is higher than men and increases with aging. On the other hand, inactivity reduces energy expenditure and causes weight gain, and thus leads to IR and increased blood glucose and dyslipidemia [19]. According to the above-mentioned evidence, older age and inactivity are important risk factors for development of MetS among CAD patients.

It seems that modification in food pattern and lifestyle among Iranian population leads to low activity and increases abdominal obesity, resulting in an increase in the rate of all coronary risk factors [30]. Since many of those at risk are not identified, further prospective studies are recommended for more clarification.

A limitation of our study was that the food habits and diet intakes in our studied population were no assessed. However, this study was performed for the first time in Zahedan (Center of Sistan and Baluchistan Province) in southeast of Iran, thus, it is the power of this research.

\section{Conclusions}

The results of the study showed that the dyslipidemia, abdominal obesity and hyperglycemia are more common risk factors for CAD; however, the combination of all components of MetS may be a secondary contributor to CAD risk. Therefore, health prevention programs are recommended to reduce the risk of CAD.

\section{Acknowledgments}

We are grateful to staff and nurses of Cardiology Department in Zahedan Imam Ali Hospital for their kind cooperation. We thank the patients who willingly participated in the study.

\section{Funding Source}

This work was supported by the Research Deputy of Zahedan University of Medical Sciences, Zahedan, Iran.

\section{Conflicts of Interest}

The authors had no conflicts of interest.

\section{Author Contributions}

Design: Farzaneh Montazerifar and Milad Mahmoodi Mozaffar. Diagnosis of CAD: Ahmad Bolouri. Data collection: Milad Mahmoodi Mozaffar. Data analysis: Farzaneh Montazerifar. Drafting of the article: Fazaneh Montazerifar and Mansour Karajibani. Interpretation of data: Farzaneh Montazerifar, Mansour Karajibani, Ahmad Bolouri, and Milad Mahmoodi Mozaffar. Revision of the final manuscript: Fazaneh Montazerifar, Mansour Karajibani, and Ahmad Bolouri.

\section{References}

1. Fiuza M. [Metabolic syndrome and coronary artery disease]. Rev Port Cardiol. 2012;31(12):779-782.

2. Hajian-Tilaki K. Metabolic syndrome and its associated risk factors in Iranian adults: A systematic review. Caspian J Intern Med. 2015;6(2):51-61.

3. Azizi F, Hadaegh F, Khalili D, Esteghamati A, Hosseinpanah F, Delavari A, Larijani B, et al. Appropriate definition of metabolic syndrome among Iranian adults: report of the Iranian National Committee of Obesity. Arch Iran Med. 2010;13(5):426-428. 
4. Veghari GH, Sedaghat M, Banihashem S, Moharloei P, Angizeh A, Tazik E, et al .The Prevalence of Metabolic Syndrome in the North of Iran. An Epidemiologic Comparative Study. J Cardiovasc Disease Res. 2015;6(4):172175.

5. Neeb ZP, Edwards JM, Alloosh M, Long X, Mokelke EA, Sturek M. Metabolic syndrome and coronary artery disease in Ossabaw compared with Yucatan swine. Comp Med. 2010;60(4):300-315.

6. Zidi W, Allal-Elasmi M, Zayani Y, Zaroui A, Guizani I, Feki M, Mourali MS, et al. Metabolic Syndrome, Independent Predictor for Coronary Artery Disease. Clin Lab. 2015;61(10):1545-1552.

7. Kaykhaei M, Hashemi M, Narouie B, Shikhzadeh A, Jahantigh M, Shirzaei E, Rezazehi B, et al. Prevalence of metabolic syndrome in adult population from zahedan, southeast iran. Iran J Public Health. 2012;41(2):70-76.

8. Sharifi F, Mousavinasab SN, Soruri R, Saeini M, Dinmohammadi M. High prevalence of low high-density lipoprotein cholesterol concentrations and other dyslipidemic phenotypes in an Iranian population. Metab Syndr Relat Disord. 2008;6(3):187-195.

9. Azizi F, Salehi P, Etemadi A, Zahedi-Asl S. Prevalence of metabolic syndrome in an urban population: Tehran Lipid and Glucose Study. Diabetes Res Clin Pract. 2003;61(1):29-37.

10. Won KB, Chang HJ, Sung J, Shin S, Cho IJ, Shim CY, Hong GR, et al. Differential association between metabolic syndrome and coronary artery disease evaluated with cardiac computed tomography according to the presence of diabetes in a symptomatic Korean population. BMC Cardiovasc Disord. 2014;14:105.

11. Third Report of the National Cholesterol Education Program (NCEP) Expert Panel on Detection, Evaluation, and Treatment of High Blood Cholesterol in Adults (Adult Treatment Panel III) final report. Circulation. 2002;106(25):3143-3421.

12. Grundy SM, Cleeman JI, Daniels SR, Donato KA, Eckel RH, Franklin BA, Gordon DJ, et al. Diagnosis and management of the metabolic syndrome: an American Heart Association/National Heart, Lung, and Blood Institute Scientific Statement. Circulation. 2005;112(17):27352752.

13. Azizi F, Khalili D, Aghajani H, Esteghamati A, Hosseinpanah F, Delavari A, Larijani B, et al. Appropriate waist circumference cut-off points among Iranian adults: the first report of the Iranian National Committee of Obesity. Arch Iran Med. 2010;13(3):243-244.

14. Arjmand G, Shidfar F, Molavi Nojoomi M, Amirfarhangi A. Anthropometric Indices and Their Relationship With Coronary Artery Diseases. Health Scope. 2015;4(3):e25120.

15. Zhang Y, Hong J, Gu W, Gui M, Chen Y, Chi Z, Wang W, et al. Impact of the metabolic syndrome and its individual components on risk and severity of coronary heart disease. Endocrine. 2009;36(2):233-238.

16. Yazdi Z, Sarreshtedari M, Tayefi MH. Prevalence of metabolic syndrome and its association factors among drivers of heavy vehicles. Ghom Uni Med Sci. 2012;5(4):68-72.
17. Malik S, Wong ND, Franklin SS, Kamath TV, L'Italien GJ, Pio JR, Williams GR. Impact of the metabolic syndrome on mortality from coronary heart disease, cardiovascular disease, and all causes in United States adults. Circulation. 2004;110(10):1245-1250.

18. Misra A, Shrivastava U. Obesity and dyslipidemia in South Asians. Nutrients. 2013;5(7):2708-2733.

19. Amarasinghe S, Sandrasegarampillai B, Arasaratnam V. Metabolic syndrome among Jaffna Tamil community, Sri Lanka. Indian J Endocrinol Metab. 2015;19(5):663-666.

20. Lopes NH, Paulitsch FS, Pereira AC, Gois AF, Gagliardi A, Garzillo CL, Ferreira JF, et al. Impact of metabolic syndrome on the outcome of patients with stable coronary artery disease: 2-year follow-up of the MASS II study. Coron Artery Dis. 2008;19(6):383-388.

21. Moussouami SI, Bouhika EJ, Nsompi F, Kayilou JMB, Mbemba F, Massamba A. Prevalence and Risk Factors of Cardiovascular Diseases in the Congo-Brazzaville Pygmies. World J Cardiovasc Diseases. 2016;6:211-217.

22. Won KB, Chang HJ, Kim HC, Jeon K, Lee H, Shin S, Cho IJ, et al. Differential impact of metabolic syndrome on subclinical atherosclerosis according to the presence of diabetes. Cardiovasc Diabetol. 2013;12:41.

23. Azizi F, Rahmani M, Emami H, Mirmiran P, Hajipour R, Madjid M, Ghanbili J, et al. Cardiovascular risk factors in an Iranian urban population: Tehran lipid and glucose study (phase 1). Soz Praventivmed. 2002;47(6):408-426.

24. Hajian-Tilaki KO, Heidari B. Prevalence of obesity, central obesity and the associated factors in urban population aged 20-70 years, in the north of Iran: a population-based study and regression approach. Obes Rev. 2007;8(1):310.

25. Ghayour-Mobarhan M, Shapouri-Moghaddam A, AzimiNezhad M, Esmaeili H, Parizadeh SM, SafarianM, et al. The relationship between established coronary risk factors and serum copper and zinc concentrations in a large Persian cohort. J Trace Elem Med Biol. 2009;23:16775.

26. Ebrahimi M, Kazemi-Bajestani SM, Ghayour-Mobarhan M, Moohebati M, Paydar R, Azimi-Nezhad M, et al. Metabolic syndrome may not be a good predictor of coronary artery disease in the Iranian population: population specific definitions are required. Sci World J. 2009;9:8696.

27. Alexander CM, Landsman PB, Teutsch SM, Haffner SM. NCEP-defined metabolic syndrome, diabetes, and prevalence of coronary heart disease among NHANES III participants age 50 years and older. Diabetes. 2003;52(5):1210-1214.

28. Esteghamati A, Abbasi M, Nakhjavani M, Yousefizadeh A, Basa AP, Afshar H. Prevalence of diabetes and other cardiovascular risk factors in an Iranian population with acute coronary syndrome. Cardiovasc Diabetol. 2006;5:15.

29. Karimi A, Marzban M, Movahedi N, Salehiomran A, Sadeghian S, Goodarzynejad H. Traditional cardiac risk factors profile in Iranian patients undergoing coronary artery bypass surgery. Acta Cardiol. 2009;64(3):371-377.

30. Gupta AK, Prieto-Merino D, Dahlof B, Sever PS, Poulter NR. Metabolic syndrome, impaired fasting glucose and obesity, as predictors of incident diabetes in 14120 hypertensive patients of ASCOT-BPLA: comparison of 
their relative predictability using a novel approach. Diabet Med. 2011;28(8):941-947.

31. Ebrahimi M, Kazemi-Bajestani SM, Ghayour-Mobarhan M, Ferns GA. Coronary artery disease and its risk factors status in iran: a review. Iran Red Crescent Med J. 2011;13(9):610-623.

32. Parizadeh SM, Moohebati M, Ghafoori F, GhayourMobarhan M, Kazemi-Bajestani SM, Tavallaie S, Azimi-Nezhad M, et al. Serum selenium and glutathione peroxidase concentrations in Iranian patients with angiography-defined coronary artery disease. Angiology. 2009;60(2):186-191.

33. Janghorbani M, Amini M. Hypertension in type 2 diabetes mellitus in Isfahan, Iran: incidence and risk factors.
Diabetes Res Clin Pract. 2005;70(1):71-80.

34. Siavash M, Sadeghi M, Salarifar F, Amini M, ShojaeeMoradie F. Comparison of body mass index and waist/ height ratio in predicting definite coronary artery disease. Ann Nutr Metab. 2008;53(3-4):162-166.

35. Hajian-Tilaki K, Heidari B, Firouzjahi A, Bagherzadeh M, Hajian-Tilaki A, Halalkhor S. Prevalence of metabolic syndrome and the association with socio-demographic characteristics and physical activity in urban population of Iranian adults: a population-based study. Diabetes Metab Syndr. 2014;8(3):170-176.

36. Sarrafzadegan N, Gharipour M, Sadeghi M, Khosravi AR, Tavassoli AA. Metabolic syndrome in Iranian elderly. ARYA Atheroscler. 2012;7(4):157-161. 